\title{
MAPSS: An Intelligent Architecture for the Pedagogical Support
}

\author{
https://doi.org/10.3991/ijet.v14i14.10362
}

\author{
Najoua Hrich ${ }^{(凶)}$, Mohamed Lazaar, Mohamed Khaldi \\ Abdelmalek Essaâdi University, Tetouan, Morocco \\ amine.najoua@gmail.com
}

\begin{abstract}
The multi-agent systems (MAS) are a part of artificial intelligence (AI), they have emerged today in the development of major e-learning platforms. Their integration has given new impetus to learning environments by the possibility of integrating new parameters (psychological, pedagogical, ergonomic) favoring a better adaptation to the learner. In addition, the multiagent approach offers the possibility to design flexible solutions based on a set of agents which are in continuous communication to accomplish the tasks entrusted to them.

In this paper, we propose a model of pedagogical support based on a coupling of ontology and multi-agent systems for a synergy of their forces and the important contribution they can make to improve the learning-teaching process.

Previous work has been the subject of theoretical foundation related to competency evaluation, and development of an ontology and an algorithm for evaluating competency. As a continuity, we present the design of Multiagent Pedagogical Support System (MaPSS) and the different scenarios of its utilization.
\end{abstract}

Keywords-Pedagogical support, assessment process, competencies, ontologies, Multiagent systems.

\section{$1 \quad$ Introduction}

The notion of pedagogical support is now very widespread. Learners who encounter difficulties during their learning process, use pedagogical support to overcome these difficulties. Even learners who are in full academic success, use it to raise their averages, to provide preparation for competitions or/and to prepare for important exams.

The use of e-learning platforms is the most adopted solution by different learners; the problem of using such systems is that all learners perform same activities whatever are their difficulties, their level, and their learning styles. Therefore, the development of an effective and efficient assessment tool is needed.

For an effective implementation of a support session, some points are unavoidable: firstly, a diagnostic assessment should be done to assess the level of learners and their knowledge, secondary, the analyzing of the outcomes of this assessment and finally, the choice of the remediation content and then its presentation according to the learners' preferences and learning styles. In this context and to ensure an efficient pedagogical 
support, we have adopted, in previous work, the semantic web ontologies by the advantages they offer in terms of complete and semantic formalization of information and we have conceived:

- A competency assessment ontology which aims to represent the evaluation of competency under an interoperable form to guarantee its reuse and sharing between different learning platforms,

- An Algorithm which describes the assessment process adopted

Our ontology describes relations between the competencies 'components, questions and the objects to evaluate. So, to validate a specific concept, the learner must answer the questions related to this concept and concepts in relation with, which leads to a more relevant assessment [1][2].

In this paper, we propose a design of pedagogical support based on multiagent approach and using the E-assessment ontology according to the proposed algorithm. The advantage to implement a MAS is due to their important contribution to improve the learning process and the possibility to design flexible solutions based on a set of agents which are in continuous communication to accomplish the tasks entrusted to them. In addition, the coupling process of ontologies and multi-agent systems allows a synergy of their forces and offers the use of our proposal with different scenarios:

- Scenario 1: MaPSS can be used as a diagnosis tool whose results can be interpreted and exploited by a human agent or IT agent, which offers the possibility of being integrated into an e-learning platform.

- Scenario 2: MaPSS can be used as a pedagogical support tool in class.

- Scenario 3: MaPSS can be used as an online pedagogical support tool.

With different scenarios, the proposed tool aims to improve the learning process for learners and teachers:

- For the learner: being exposed to a content that suits his profile and that targets the knowledge he lacks, by using an optimal pedagogical approach that keeps the learner in his proximal development zone, he is more likely to perceive his learning as relevant and challenged and he focuses on notions he does not master and he devotes all his energy to the learning he needs.

- For the tutor/teacher: The tool provides precious information that allows him to identify what knowledge and skills he would develop with his learners. Thanks to the collected data, this tool allows him to know if the invested efforts in training report as desired or it is necessary to adjust the shot.

\section{Design of MaPSS}

From a pedagogical point of view, the proposed architecture for MaPSS tool is based on the fact that the pedagogical support is a process who necessitate two principal tasks:

- The diagnostic of knowledge, the analyze and the treatment of its outcomes. 
- The proposition of remedial situations according to learner styles and preferences $[1,2,3,4]$.

The tasks of supporting process are shown in figure 1.

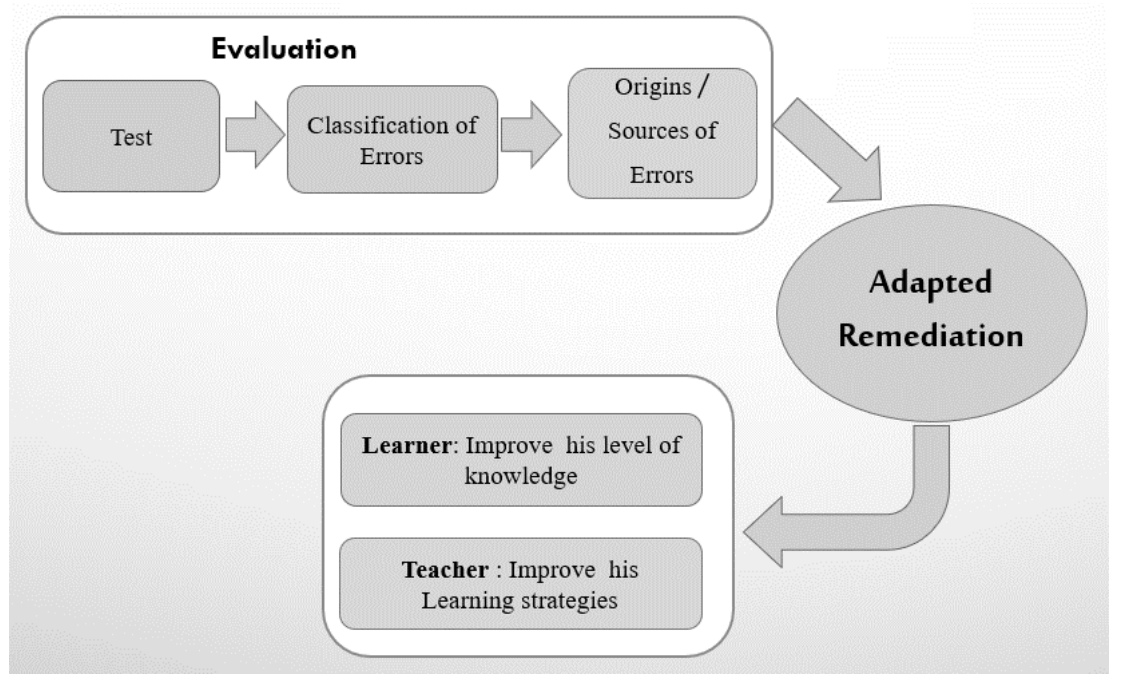

Fig. 1. The process of pedagogical support

Those tasks can be executed by one agent (tutor), and it is possible to distribute them between different agents: each task is trusted to a specific agent. In this optic and to offer different possibility of using our tool and to achieve the main objective of MaPSS which is offering a pedagogical support that respond to the specific cognitive needs for each learner using our system, this task is subdivided on two sub-tasks distributed between two agents: EvalAgent for the diagnostic task and support agents to determine the adapted remedial situations.

From a technical and informatic point of view, we have opted for the agent technology and in particular we have adopted multi-agent systems because -The use of multiagent systems provides the reuse, the portability, the robustness, the interoperability of other systems and the ease of maintenance and the extension or reduction of the architecture as required: agents can dynamically add or remove. -Multi-agent systems are ideal systems to represent problems with multiple methods of resolution, multiple perspectives [5][6]. They have the advantages of distributed and concurrent resolution of problems such as scalability, speed if multiple agents can work in parallel, they also inherit the conceivable benefits of artificial intelligence.

Figure 2 represents an architecture which integrates EvalAgent and SupportAgent interacting with an interface agent (IA) and a SyleAgent (SA) and an ontology whose roles will be detailed in the next sections. 


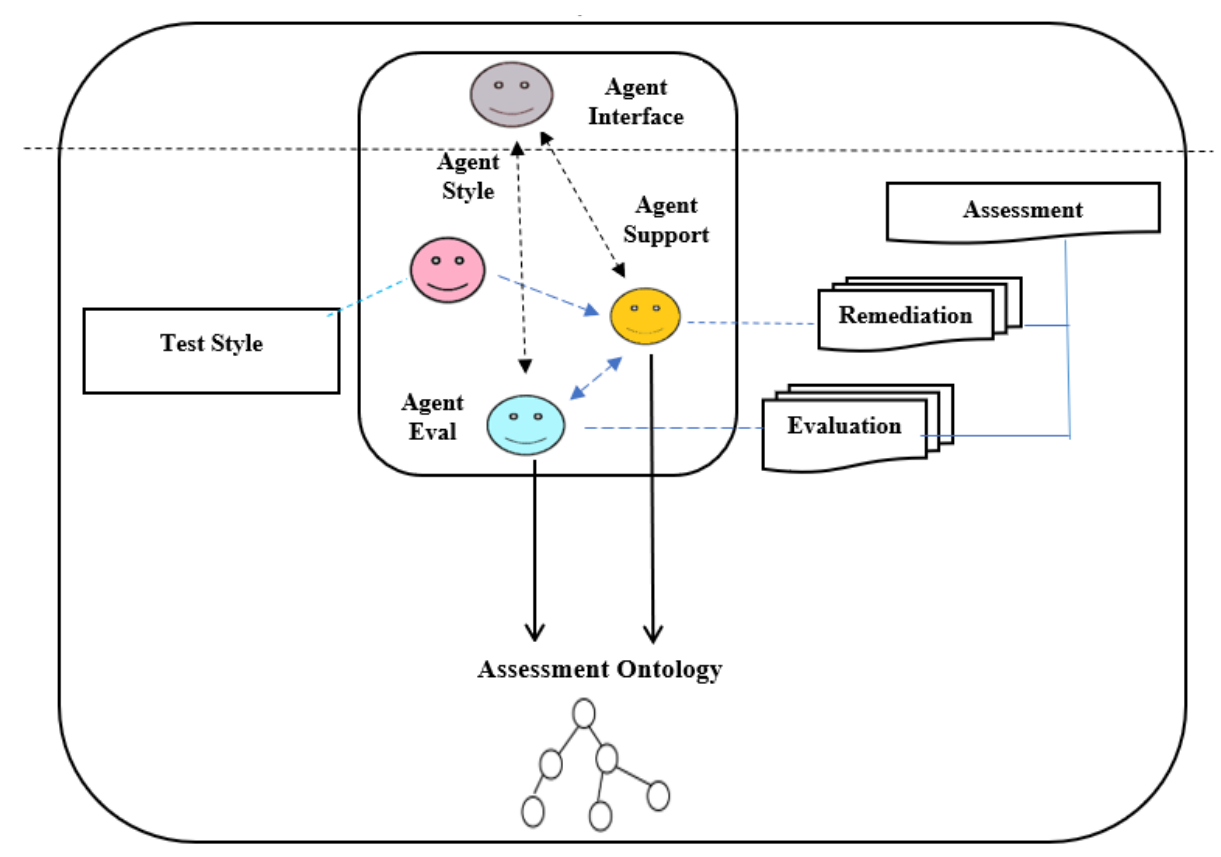

Fig. 2. Architecture of MAPSS

\subsection{Description of agents}

The architecture we propose is based on flexible modules (agents) that allow interaction with the learner to offer an efficient supporting and it's composed of the following agents:

Interface agent (IA): It is an Adaptive Agent who according to the profile of the human user: Teacher or learner changes his appearance and functionalities

- For the learner: The IA allows him to register for a support session, to do a test and remediation if necessary.

- For the teacher: Offers the possibility of enriching the basis of remediation activities and tests.

Style agent (SA): It is responsible for determining the learning styles of the learner and its communication to the support agent.

Evaluateur agent (EvalAgent): This agent is responsible for presenting the evaluation test for each concept, and communicating the results to the support agent. The assessment is presented according to the ontology (assessment ontology figure 3).

Support agent (SpA): According to the results communicated by EvalAgent and $\mathrm{SA}$, the SpA decides on the type/content of the activity to be presented to the learner if it requires remediation and then sends the message of continuing test to Evalagent. If the learner doesn't need remediation, the message continuing test is sent directly to EvalAgent. 


\section{$2.2 \quad$ Assessment ontology}

To present the relationship between the test questions and the concepts to evaluate and between those concepts and their remedial activities contents, we adopt, in our approach, the semantic web ontologies by the advantages they offer in terms of complete and semantic formalization of information. For this, we have conceived a competency assessment ontology which aims to represent the evaluation of competence under an interoperable form to guarantee its reuse and sharing between different learning platforms.

Our ontology describes relations between the competencies 'components, questions and the objects to evaluate and remedial activities for concepts and their mobilization.

For the implementation of the ontology, we used protege because it is a free opensource ontology editor and framework for building intelligent systems and very popular in semantic web and computer research [7]. Protege provides a Java API for application developers to access and modify all aspects of Protege knowledge bases and its user interface. Protege stores ontologies in many different formats including relational databases, UML, XML, RDF, and OWL language; which meets our needs in terms of the development of our system based on the agent technology.

The following figures show respectively the classes hierarchy, an overview of the owl file representing the instantiation of the Assessment ontology and its OntoGraf. This ontology is used by instructional designers, the task of instructional designers is central, because they are required to state much more finely the different components of a competency and the remedial activities related to each component $\&$ their mobilization in order to facilitate to the teachers to diagnose or to create support activities and the learner to explore their needs and expectations.

\begin{tabular}{|c|c|}
\hline Class hierarchy: & 므미 \\
\hline \begin{tabular}{|l|l|l|}
+5 & $\varnothing$ \\
\end{tabular} & Asserted $\mathbf{V}$ \\
\hline $\begin{array}{l}\text { owl:Thing } \\
\text { unit } \\
\text { Concept } \\
\text { Acquisition_of_Concepts } \\
\text { Mobilizations_of_concept } \\
\text { Questions_of_ressource } \\
\text { RemediationActivity }\end{array}$ & \\
\hline
\end{tabular}

Fig. 3. The Classes Hierarchy of Assessment Ontology 


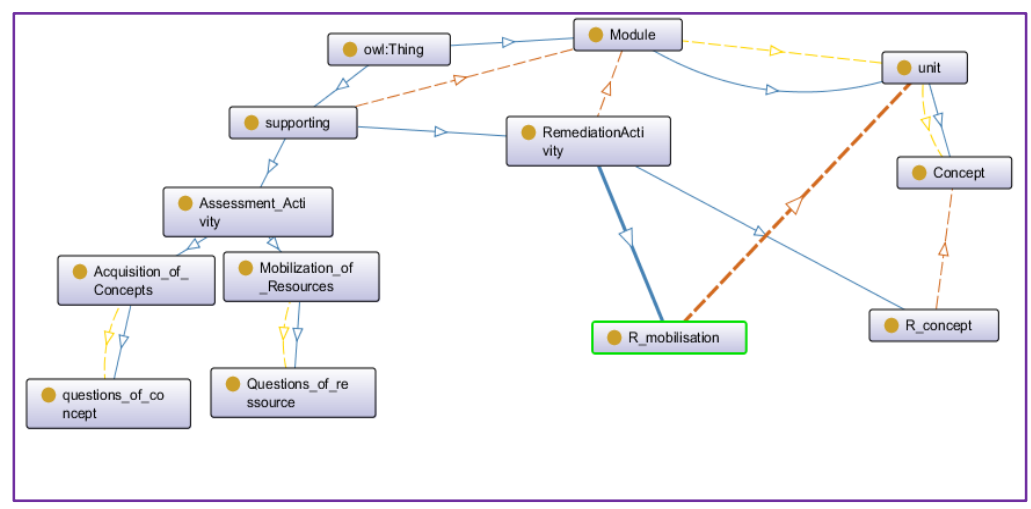

Fig. 4. The OntoGraf of Assessment Ontology

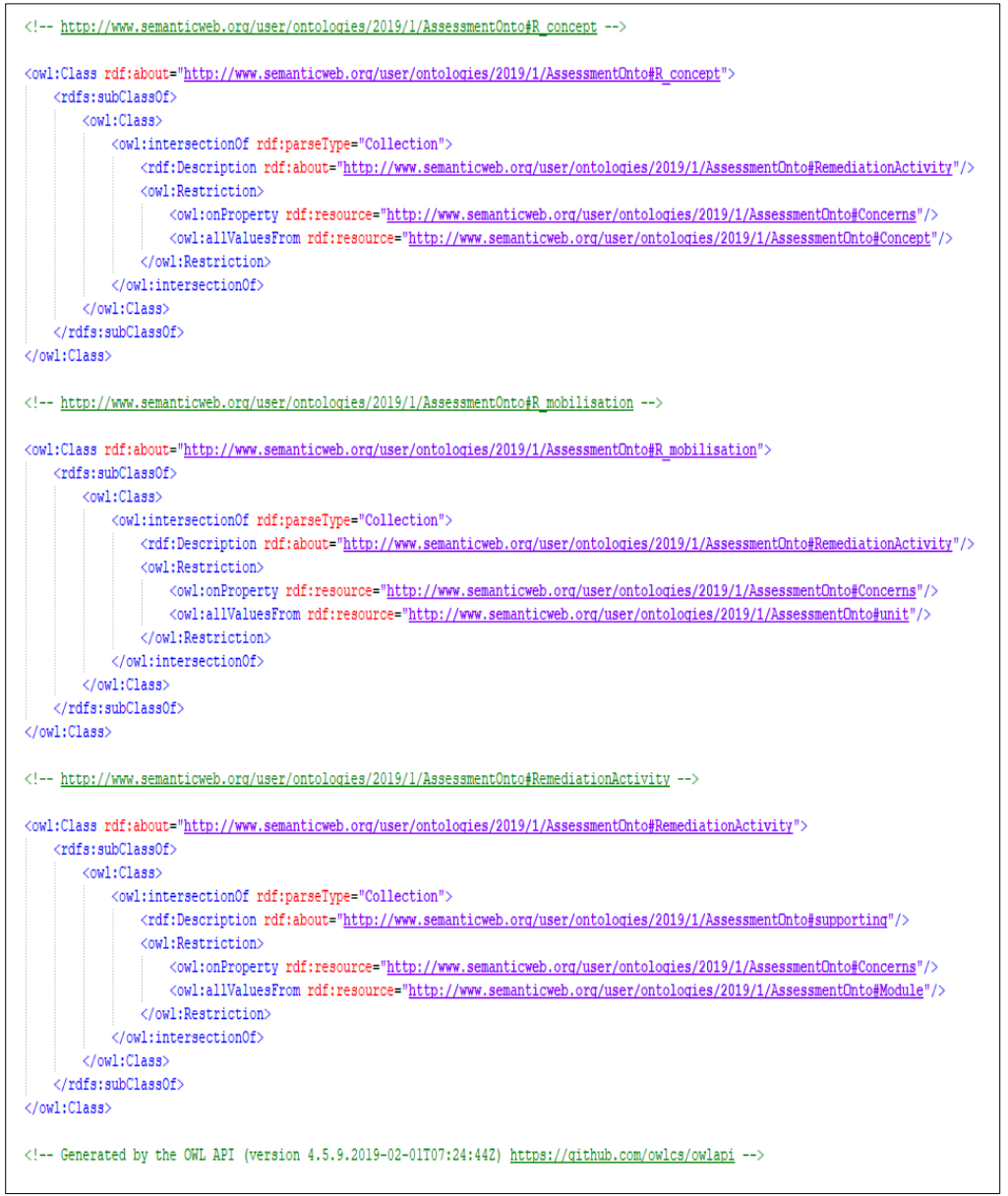

Fig. 5. Overview of The Owl File of Assessment Ontology 


\section{Description of MaPSS Operation}

To describe the operating principle of MaPSS system we use behavior diagrams. Each diagram contains the name of the agent, the role it plays in a specified scenario [8]. The arrows represent the interactions between the agents. An agent is represented by a white square, a human actor by a gray square. This type of notation is particularly used to the representation of interactions in a multi-agent system composed of artificial and human agents.

In this section, we illustrate how our system operates through the following scenarios, for each scenario we present a behavior diagrams for opening a session and for the test administration.

\subsection{Scenarios of MaPSS uses}

Scenario 1: MaPSS is used for diagnostics purposes In this scenario, SA and SpA are inactive. When a new learner logs in to the system, IA recovers information about it and checks its access rights. Afterward, EvalAgent is informed of his presence and propose to him the diagnostic test of the targeted competency using the Assessment Ontology and the appropriate pedagogical rules. Finally, a cognitive report is represented to the learner.

Opening a session

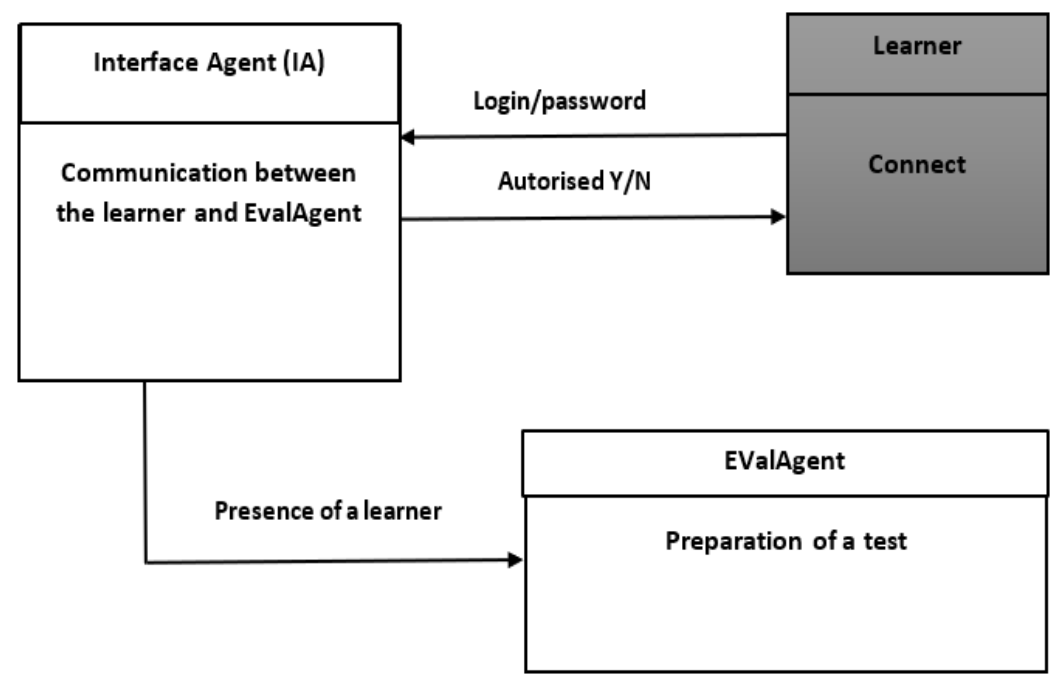

Fig. 6. Diagram of Opening a session by a Learner 


\section{Test administration}

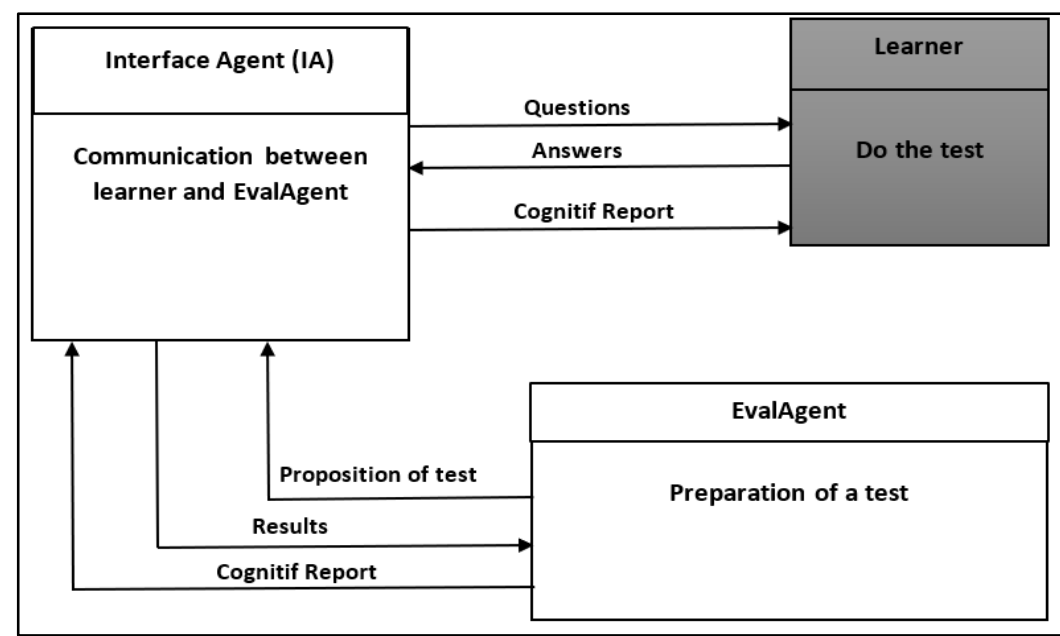

Fig. 7. Diagram of test administration by the agents

Scenario 2: MaPSS is used by a teacher with a class group for diagnostic purposes in this scenario, the SA and the SpA are inactive. The teacher creates the class groups. When a new learner logs to the system The IA retrieves information about it, checks its access rights. Then, IA retrieves information about it and checks its access rights. Afterward, EvalAgent is informed of his presence and propose to him the diagnostic test of the targeted competency using the Assessment Ontology and the appropriate pedagogical rules. Finally, a cognitive report is shown to each learner, another report can be visualized by the teacher.

\section{Opening a session}

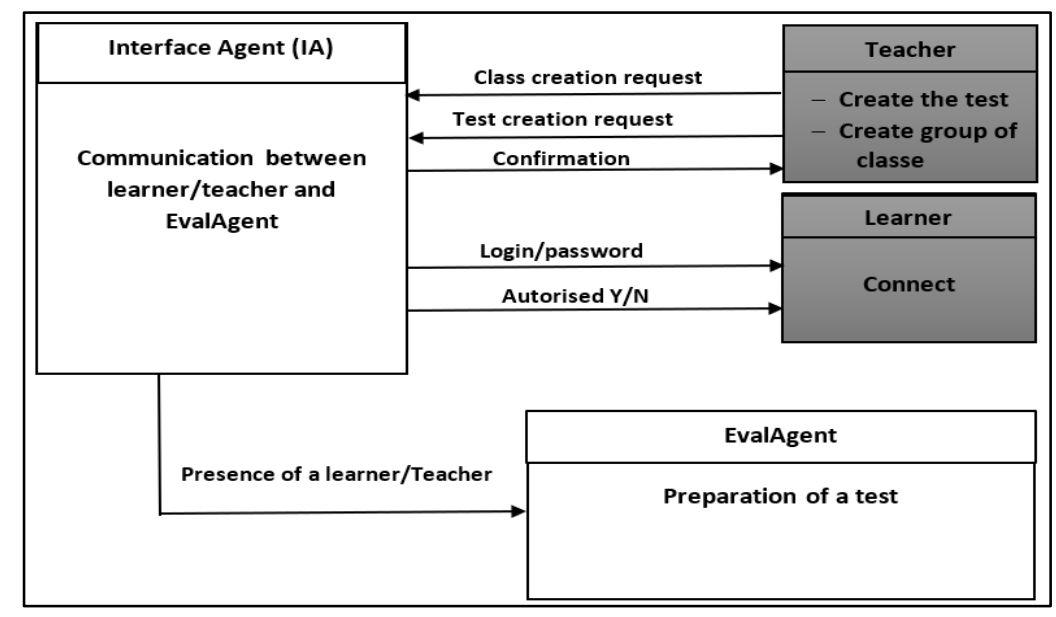

Fig. 8. Diagram of the Opening session by a Learner/ Teacher 


\section{Test administration}

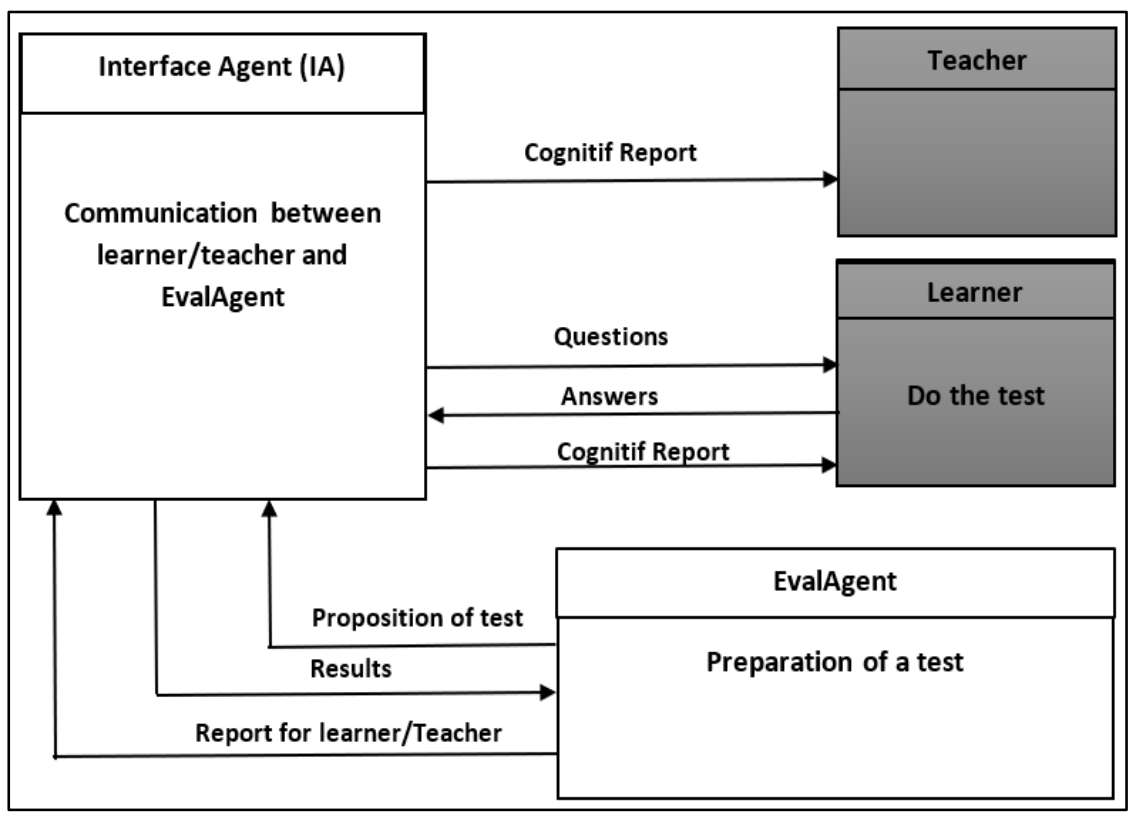

Fig. 9. Diagram of administration of test by the agents

Scenario 3: MaPSS is used for pedagogical support in this scenario, all agents are active, when a learner logs to the system, the IA retrieves information about him and checks his access rights

If it is a new learner, SA presents a test to determine his learning styles. If the learner is already registered, SA retrieves information about him and communicate them to SpA.

When finishing its task, the SA send a msg to EvalAgent to begin the diagnostic of concepts. At the end of each diagnostic, results are transferred to SpA. With the required information from SA, EvalAgent and the assessment ontology, SpA decides about the adapted remediation to deliver the more efficient support for each learner.

In this case, MaPSS is used as a tool that fulfills the different tasks of the pedagogical support process: diagnostic, analyses of results and remediation. It could play the role of a teacher in a support session concerning a learning module.

\section{Opening a session}

In this scenario, the diagram of opening a session is the same presented in figure 4. 


\section{Administration of pedagogical support}

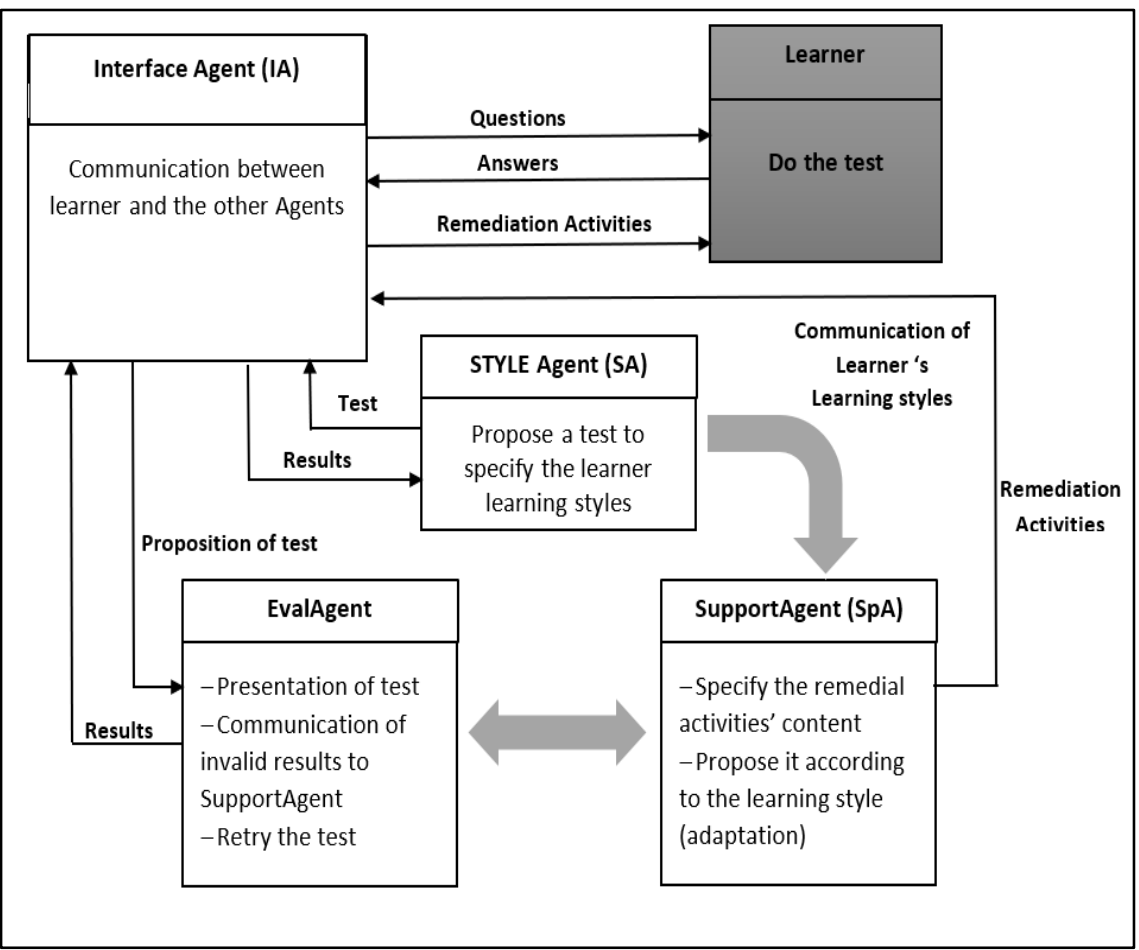

Fig. 10. Diagram of administration of support by the agents

\subsection{Diagnostic reports}

Cognitive reports mentioned in previous figures present feedbacks for learners and teachers. According to Hattie [9], Feedback refers to the process of securing information enabling change through adjustment or calibration of efforts in order to bring a person closer to a well-defined goal.

In order to achieve the main goal of the pedagogical support, MaPSS present two types of report which contain useful feedbacks for both teachers and learners: Learner Report and Teacher Report

\section{Learner report}

- The acquired \& the non-acquired concepts

- The capacity of their mobilization to apprehend a situation and to resolve it.

- Other alternatives to solve the task

- An indication of tracks of deepening / prolongation.

Teacher report: When learners make erroneous reasoning, the teacher can ask/assess if the strategies he used at his teaching sessions are optimal or not; and what he has to change for remediation. 
- The rate of learners who acquired / not acquired each concept

- The rate of learners who are able to mobilize concepts for resolving complex situations.

- List of concepts which need remediation.

\section{Conclusion}

The assessment task is an important step inside the e-learning process because gives convenient feedback to all participants in the process and helps to improve the learningteaching experience.

In previous works, we emphasized the role of the assessment by putting it at the center of the e-learning process and defining the important factors to the main elements that participate in this process: the specification of learner's source of errors and overcome them in a real time is the key of any adaptation. The adaptation must first take into account the content of the activity to present for the learner and then the way it will be presented (according to the learning style); we have presented a novel view of assessment which could be exploited into adaptive e-learning systems. The proposed tool requires collaboration between different agents (IT or human) and allows to specify for each learner the adapted content and then the way to present it according to their preferences and their learning styles.

In a supporting session, the assessment of knowledge presents a main task which must give relevant information to determine learners' strengths and weaknesses. The presentation of remedial activities according to learners' learning styles allows an efficient adaptation.

In this paper we presented an adaptive architecture based on ontologies, multi-agent systems for the presentation of the pedagogical support in its principal tasks: assessment of knowledge, analyze of results and adapt remediation. We have designed an ontology to present the relationship between the test questions and the concepts to evaluate and between those concepts and their remedial activities contents. Also, we have presented the operation of each agent (IT or human) of our system and the principle of collaboration between them.

The proposed system can be used with different scenarios depending on the excepted purposes by the users.

Next works are the implementation of a prototype in a specific domain and its experimentation with different scenarios.

\section{$5 \quad$ References}

[1] Hrich, N., Lazaar, M., Khaldi, M, 2019. Improving Cognitive Decision-Making into Adaptive Educational Systems through A Diagnosis Tool based on competency approach (IJET), (In editing) https://doi.org/10.3991/ijet.v14i07.9870

[2] Hrich, N., Lazaar, M., Khaldi, M, 2017. A model for pedagogical supporting based on competencies evaluation and ontologies, International Research Journal of Computer Science (IRJCS) 
[3] Hrich, N., Lazaar, M., Khaldi, M., 2015. Proposal of a new approach for supplementary tutoring online. International Journal of Engineering Science and Innovative Technology (IJESIT), 4, 35-40.

[4] Hrich, N., Lazaar, M., Khaldi, M., 2015. Integration of a Diagnostic Module into an Intelligent Tutoring System. International Research Journal of Computer Science (IRJCS), 2, 1117.

[5] Brahim Chaib-draa, Agents et systèmes multiagents, Notes de cours, Département d'informatique, Faculté des sciences et de gnie, Université Laval, Québec, Novembre 1999 https://doi.org/10.17660/ejhs.2017/82.2.2

[6] J. Ferber. Les systèmes multi-agents, Inter Editions, Paris, 1995

[7] https://protege.stanford.edu/

[8] Moulin, B., Brassard, b; A Scenario-Based Design Method and an Environment for the Development of Multiagent Systems. In C. Zang and D. Lukose(editor), Proceedings of the First Australian Workshop on DAI, Volume LNAI 1087, Springer Verlag, 1995.

[9] Hattie et Yates, "Visible learning and the science of how we learn", Routledge, NY, 2014, p.66

\section{Authors}

Najoua Hrich is with the Computer lab, operational research, and applied statistics, Abdelmalek Essaadi University, Faculty of Sciences, Tetouan, Morocco (e-mail: amine.najoua@gmail.com).

Mohamed Lazaar with the Computer lab, operational research, and applied statistics, Abdelmalek Essaadi University, ENSA, Tetouan, Morocco (e-mail: lazaarmed @.gmail.com).

Mohamed Khaldi is with the Computer lab, operational research, and applied statistics, Abdelmalek Essaadi University, Ecole Normale Superieure Tetouan, Morocco (e-mail: medkhaldi@yahoo.fr).

Article submitted 2019-02-23. Resubmitted 2019-04-13. Final acceptance 2019-04-15. Final version published as submitted by the authors 